\title{
Síndrome de Budd-Chiari: etiología, manejo y resultados en una cohorte de 35 pacientes del Hospital Pablo Tobón Uribe
}

\section{Budd-Chiari Syndrome: Etiology, management, and outcomes in a study of 35 patients at the Hospital Pablo Tobón Uribe}

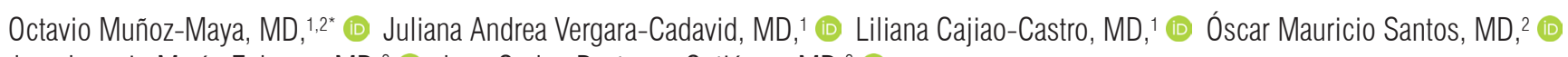
Juan Ignacio Marín-Zuluaga, MD, ${ }^{2}$ (i) Juan Carlos Restrepo-Gutiérrez, MD. ${ }^{2}$ (C)

\begin{abstract}
GACCESO ABIERTO
Citación:

Muñoz-Maya 0, Vergara-Cadavid JA, CajiaoCastro L, Santos OM, Marín-Zuluaga JI,

Restrepo-Gutiérrez JC. Síndrome de Budd-Chiari: etiología, manejo y resultados en una cohorte de 35 pacientes del Hospital Pablo Tobón Uribe. Rev Colomb Gastroenterol. 2020;35(3):280-286 https://doi.org/10.22516/25007440.431

Grupo de Gastrohepatología y Facultad de Medicina, Universidad de Antioquia; Medellín, Colombia.

2 Grupo de Gastrohepatología y Facultad de Medicina. Universidad de Antioquia, Hospital Pablo Tobón Uribe; Medellín, Colombia.

*Correspondencia: Octavio Muñoz-Maya, MD octavio.g.munoz@gmail.com
\end{abstract}

Fecha recibido: $\quad 17 / 07 / 19$ Fecha aceptado: 03/10/19

\section{Resumen}

El síndrome de Budd-Chiari (Budd-Chiari syndrome, BCS) es una entidad rara, definida por la obstrucción del flujo venoso del hígado y corresponde a una condición heterogénea tanto en presentación como en manejo terapéutico. A continuación, presentamos un estudio retrospectivo de pacientes con diagnóstico de BCS, atendidos por el grupo de hepatología del el Hospital Pablo Tobón Uribe. Los resultados se expresan como proporciones en las variables categóricas y como medias y rangos para las variables continuas. La edad promedio de presentación fue de 37,7 años y no hubo preponderancia por el género. Los principales síntomas fueron dolor abdominal $(91,4 \%)$, ascitis $(71,4 \%)$ y hepatomegalia $(60 \%)$. Asimismo, 11 pacientes $(31,4 \%)$ presentaron hipercoagulabilidad primaria, mientras que en $7(20 \%)$ se encontró algún síndrome mieloproliferativo. Además, 6 (17,1\%) tenían neoplasias extrahepáticas: 3 con compresión mecánica y 3 por hipercoagulabilidad asociada; solo en 1 caso se documentaron membranas en la vena cava inferior. $Y$ en 5 casos no fue posible determinar la causa de trombosis. De igual forma, 32 pacientes $(91,4 \%)$ recibieron anticoagulación como primer manejo; solo 4 sujetos fueron remitidos a procedimientos: 2 a angioplastia, 1 a derivación portosistémica intrahepática transyugular (Transjugular Intrahepatic Portosystemic Shunt, TIPS) y 1 a trasplante ortotópico hepático $(\mathrm{TOH})$. Luego de ello, $17(48,5 \%)$ recibieron procedimientos invasivos como segunda línea: 8 (22,8 \%) se condujeron a angioplastia y endoprótesis (stent), 5 (14,29 \%) a TIPS y 4 necesitaron TOH. Así, los 6 pacientes que se llevaron a TIPS no necesitaron trasplante hepático. El BCS continúa siendo una condición poco frecuente que afecta la salud de personas jóvenes, tanto hombres como mujeres, con una presentación clínica heterogénea. Sin embargo, en la mayoría de los casos ocurre por una causa desencadenante clara, entre las cuales predominan las trombofilias y los síndromes mieloproliferativos. El uso de medidas terapéuticas invasivas, especialmente de la selección temprana de los enfermos que se benefician de TIPS, ha cambiado la historia natural y el pronóstico de este grupo de pacientes.

\section{Palabras clave}

Síndrome de Budd-Chiari, trombosis de la vena hepática, trombosis.

\section{Abstract}

Budd-Chiari syndrome (BCS) is a rare disorder characterized by the obstruction of the veins of the liver. Both its presentation and therapeutic management are heterogenous. The following is a retrospective study of patients diagnosed with BCS treated by the hepatology service at the Hospital Pablo Tobón Uribe. The results of the categorical variables are expressed as proportions and the continuous variables as means and ranges. The average age of onset of this disease was 37.7 years and it was not predominant in neither sex. The most common symptoms were abdominal pain $(91.4 \%)$, ascites $(71.4 \%)$ and hepatomegaly $(60 \%)$. Out of 35 patients, $11(31.4 \%)$ had primary hypercoagulability, 7 (20\%) had some myeloproliferative disorder, 6 $(17.1 \%)$ had extrahepatic malignancies -3 with mechanical compression and 3 due to associated hypercoagulability - and 1 case had a membranous obstruction of the inferior vena cava. 32 patients $(91.4 \%)$ received anticoagulation as first-line therapy and only 4 were taken to surgery: 2 to angioplasty, 1 to transjugular intrahepatic portosystemic shunt (TIPS) and 1 to orthotopic liver transplantation (OLT). On the other hand, 17 $(48.5 \%)$ individuals underwent invasive procedures as a second-line therapy, of which $8(22.8 \%)$ underwent 
angioplasty and stenting, 5 (14.29\%) underwent TIPS and 4 needed OLT. The 6 patients who were taken to TIPS did not need liver transplants. Budd-Chiari syndrome remains a rare condition affecting the health of young men and women alike, with a heterogeneous clinical presentation but, in most cases, with a clear trigger where thrombophilic and myeloproliferative disorders predominate. The use of invasive therapeutic measures, especially the early selection of patients who may benefit from TIPS, has changed the natural course and prognosis of this group of patients.

\section{Keywords}

Budd-Chiari Syndrome; Hepatic Vein Thrombosis; Thrombosis.

\section{INTRODUCCIÓN}

El síndrome de Budd-Chiari (BCS) es una entidad rara, definida por la obstrucción del flujo venoso del hígado. Dicha obstrucción puede localizarse desde las venas hepáticas, o en cualquier parte de su trayecto, hasta la vena cava inferior. Se estima una incidencia mundial de esta condición de 1 caso por cada 2,5 millones de personas por año (1).

Aunque clásicamente este síndrome se ha definido por la triada de ascitis, dolor abdominal y hepatomegalia, en la práctica clínica es evidente que corresponde a una condición heterogénea en su presentación y manejo. Los pacientes pueden tener trombosis asociada a una trombofilia primaria o un fenómeno mecánico que desencadene la obstrucción y, en muchos casos, múltiples factores precipitantes. Las opciones terapéuticas incluyen manejo farmacológico con anticoagulación y diuréticos o procedimientos invasivos por radiología, como trombólisis, angioplastia o derivación portosistémica intrahepática transyugular (Transjugular Intrahepatic Portosystemic Shunt, TIPS) y manejo quirúrgico en el caso de la derivación portosistémica o el trasplante de hígado (2).

El conocimiento acerca de este grupo de pacientes está basado en series de caso pequeñas, y en los últimos años se han tratado de homogenizar los criterios diagnósticos, indicadores pronósticos y modalidades terapéuticas paso a paso, basadas en las posibilidades de éxito (3).

A continuación, presentamos una serie de casos retrospectiva, con el objetivo de describir las características clínicas, bioquímicas, imagenológicas, plan de manejo y desenlace de los pacientes de todas las edades con diagnóstico de BCS, atendidos en el Hospital Pablo Tobón Uribe.

\section{MATERIALES Y MÉTODOS}

Se trata de una cohorte retrospectiva, cuya población de estudio está conformada por pacientes con diagnóstico de BCS, atendidos por el grupo de hepatología del Hospital Pablo Tobón Uribe de Medellín, entre enero de 2010 y noviembre 2018.
Así pues, se definió el BCS como la obstrucción del tracto de salida venoso hepático, independiente de la causa, y que puede localizarse en las venas hepáticas, en la vena cava inferior o en ambas. Esta obstrucción puede confirmarse mediante pruebas de imagen (ecografía Doppler, tomografía computarizada, resonancia nuclear magnética contrastada o venografía).

Se excluyeron aquellos pacientes que tuvieran una enfermedad microvascular (síndrome de obstrucción sinusoidal), falla cardíaca o historia previa de trasplante hepático. En todos los pacientes, se analizaron variables demográficas generales, comorbilidades, etiología de la enfermedad hepática, forma de presentación, gravedad de la patología -clasificada por la escala de MELD (Model for End-stage Liver Disease) y por el puntaje de Rotterdam-, características de laboratorio, variables imagenológicas, manejo realizado, complicaciones y sobrevida general.

Los resultados se expresan como variables continuas, que se describen como medianas y rangos intercuartílicos (RIC), y variables categóricas, las cuales se explican como números absolutos y porcentajes.

\section{RESULTADOS}

Durante el período de estudio se documentaron 35 pacientes que se ajustaron a los criterios de inclusión. La distribución por género fue muy similar: 18 pacientes hombres $(51,43 \%)$ y 17 mujeres $(48,57 \%)$. El promedio de edad fue de 39 años (20-46 años) (Tabla 1).

Dentro de los factores de riesgo documentados antes de la presentación, 7 pacientes $(20 \%)$ tenían algún tipo de neoplasia; $6(17 \%)$ presentaban antecedentes de consumo de anticonceptivos orales; $4(11,4 \%)$ tenían historia de hipercoagulabilidad primaria documentada; 2 se manifestaron en circunstancias asociadas al período de embarazo/ puerperio; 2 contaban con historia de sarcoidosis; 2 estaban asociados a una infección local o sistémica, y en 12 casos $(34,29 \%)$ no se documentó ningún factor de riesgo. Es importante resaltar que 4 pacientes $(11,4 \%)$ tenían más de 1 factor de riesgo asociado (Tabla 2). Respecto a 
Tabla 1. Características clínicas

\begin{tabular}{|c|c|c|c|c|}
\hline & Total & Agudo & Subagudo & Crónico \\
\hline Número total & 35 & 13 & 12 & 10 \\
\hline \multicolumn{5}{|l|}{ Datos demográficos } \\
\hline Masculino & $18(51,4 \%)$ & $7(53,9 \%)$ & $6(50 \%)$ & $5(50 \%)$ \\
\hline Femenino & $17(48,6 \%)$ & $6(46,2 \%)$ & $6(50 \%)$ & $5(50 \%)$ \\
\hline Edad & $39(20-46)$ & $21(18,5-37)$ & $43,5(32,5-55,3)$ & $42,5(32,5-50,5)$ \\
\hline IMC & $23(20-26,6)$ & $23(19,7-25,8)$ & $21,6(19,2-25,1)$ & $26(22,1-29,6)$ \\
\hline \multicolumn{5}{|l|}{ Duración de los síntomas } \\
\hline$<1$ mes & $20(57,1 \%)$ & $13(100 \%)$ & $7(58,3 \%)$ & 0 \\
\hline $1-6$ meses & $8(22,9 \%)$ & 0 & $5(41,7 \%)$ & $3(30 \%)$ \\
\hline$>6$ meses & $6(17,1 \%)$ & 0 & 0 & $6(60 \%)$ \\
\hline Asintomático & $1(2,9 \%)$ & 0 & 0 & $1(10 \%)$ \\
\hline \multicolumn{5}{|l|}{ Tipo } \\
\hline Primario & $32(91,4 \%)$ & $12(92,3 \%)$ & $10(83,3 \%)$ & $10(100 \%)$ \\
\hline Secundario & $3(8,6 \%)$ & $1(7,7 \%)$ & $2(16,7 \%)$ & 0 \\
\hline \multicolumn{5}{|l|}{ Lugar de obstrucción } \\
\hline Alguna vena hepática & $7(20 \%)$ & $3(23,1 \%)$ & $2(16,7 \%)$ & $2(20 \%)$ \\
\hline Ambas venas hepáticas & $15(42,9 \%)$ & $7(53,84 \%)$ & $6(50 \%)$ & $2(20 \%)$ \\
\hline Vena cava & $2(5,7 \%)$ & 0 & 0 & $2(20 \%)$ \\
\hline Vena cava y vena hepática & $11(31,4 \%)$ & $3(23,07 \%)$ & $4(33,33 \%)$ & $4(40 \%)$ \\
\hline \multicolumn{5}{|l|}{ Manifestaciones clínicas } \\
\hline Edema de MI & $23(65,7 \%)$ & $10(76,9 \%)$ & $7(58,33 \%)$ & $6(60 \%)$ \\
\hline Ascitis & $25(71,4 \%)$ & $10(76,9 \%)$ & $8(66,66 \%)$ & $7(70 \%)$ \\
\hline Ictericia & $6(17,1 \%)$ & $4(30,8 \%)$ & $1(8,33 \%)$ & $1(10 \%)$ \\
\hline Circulación colateral & $3(8,6 \%)$ & 0 & $1(8,33 \%)$ & $2(20 \%)$ \\
\hline Fiebre & $11(31,4 \%)$ & $8(61,53 \%)$ & $2(16,66 \%)$ & $1(10 \%)$ \\
\hline Dolor abdominal & $32(91,4 \%)$ & $12(92,30 \%)$ & $11(91,66 \%)$ & $9(90 \%)$ \\
\hline Sangrado gastrointestinal & $4(11,4 \%)$ & $2(15,38 \%)$ & $1(8,33 \%)$ & $2(20 \%)$ \\
\hline Encefalopatía hepática & $3(8,6 \%)$ & $3(23,07 \%)$ & 0 & 0 \\
\hline Esplenomegalia & $8(22,9 \%)$ & $2(15,38 \%)$ & $2(16,66 \%)$ & $5(50 \%)$ \\
\hline Hepatomegalia & $21(60 \%)$ & $9(69,23 \%)$ & $5(41,66 \%)$ & $7(70 \%)$ \\
\hline \multicolumn{5}{|l|}{ Bioquímica } \\
\hline $\mathrm{Cr}$ & $0,9(0,7-1,2)$ & $1,3(0,79-1,99)$ & $0,77(0,62-1,13)$ & $0,9(0,69-0,98)$ \\
\hline BT & $1,5(0,8-2,58)$ & $1,9(0,82-3,15)$ & $1,35(0,8-2,1)$ & $1,36(0,82-2,58)$ \\
\hline INR & $1,36(1,14-1,8)$ & $1,8(1,29-1,95)$ & $1,4(1,15-1,5)$ & $1,16(1,0-1,27)$ \\
\hline Rotterdam & $1,13(0,25-1,21)$ & $1,16(0,6-2,15)$ & $1,12(0,3-1,2)$ & $1,1(0,2-1,17)$ \\
\hline MELD & $13(9-18)$ & $18(15-27,5)$ & $12,5(9,3-17,5)$ & $9(8,5-12,3)$ \\
\hline Mortalidad & $8(22,9 \%)$ & $4(30,8 \%)$ & $4(33,3 \%)$ & 0 \\
\hline Días transcurridos hasta muerte & $43,5(12,25-95)$ & $12,5(5,25-26,5)$ & $94(65-107,25)$ & 0 \\
\hline
\end{tabular}

IMC: índice de masa corporal; MI: miembros inferiores; INR: índice internacional normalizado (International Normalized Ratio); Cr: creatinina; MELD: Model for End-stage Liver Disease 
Tabla 2. Factores de riesgo identificados

Factores de riesgo

n (\%)

Cualquier trombofilia primaria

Mutación del factor $\mathrm{V}$ Leiden

$1(2,9)$

Mutación del gen de la protrombina

$0(0)$

Deficiencia de proteína $\mathrm{C}$

$1(2,9)$

Deficiencia de proteína S

$0(0)$

Deficiencia de antitrombina

$0(0)$

Cualquier trombofilia adquirida

Síndrome antifosfolípido

$4(11,43)$

Hiperhomocisteinemia

$0(0)$

Hemoglobinuria paroxística nocturna

$0(0)$

Asociada a neoplasia no hematológica

$6(17,1)$

Linfoma

Síndrome mieloproliferativo o mutación del JAK2

Policitemia rubra vera

$4(11,4)$

Trombocitosis esencial

$4(11,4)$

Mielofibrosis

$1(2,9)$

No clasificada

$1(2,9)$

Oculta

$1(2,9)$

Factores hormonales

Anticonceptivos orales

$6(17,1)$

Embarazo/puerperio

Condiciones sistémicas

Enfermedades del tejido conectivo

Sarcoidosis

$2(5,7)$

Infecciones

$4(11,4)$

Vasculitis

Sin causa documentada

$5(14,3)$

la forma de presentación clínica y los 3 síntomas reportados de forma clásica en la semiología, 32 pacientes (91\%) tuvieron dolor abdominal, 25 (71\%) presentaron ascitis, mientras que la hepatomegalia clínica fue evidente en 21 pacientes $(60 \%)$ (Figura 1$)$.
Solo 13 pacientes $(37,14 \%)$ se presentaron con BCS agudo, de los cuales 3 desarrollaron criterios de falla hepática aguda. El resto de los enfermos se presentaron de forma subaguda o crónica (Tabla 1). En cuanto a las causas documentadas después de la presentación, 11 pacientes (31,4 \%) tenían hipercoagulabilidad primaria, mientras que en 7 casos $(20 \%)$ se documentó algún tipo de síndrome mieloproliferativo. Además, 6 pacientes $(17,1 \%)$ tenían neoplasias extrahepáticas, 3 se presentaron con una compresión mecánica de las venas suprahepáticas y otros 3 cursaban con una hipercoagulabilidad asociada; solo en 1 caso se documentaron membranas en la vena cava inferior.

Asimismo, se halló que en 1 (2,8\%) de los pacientes el BCS fue secundario a una infección activa (trombosis séptica), mientras que $4(11,42 \%)$ de ellos se clasificaron en otras condiciones como el síndrome hipereosinofílico $(n=1)$, BCS secundario a trombosis de catéter tunelizado $(n=1)$ y sarcoidosis hepática $(\mathrm{n}=2)$. En los 5 casos $(14,28 \%)$ restantes no se documentó una causa desencadenante (Tabla 2). De forma global, 32 casos $(91,4 \%)$ se catalogaron como primarios asociados a una trombosis y solo 3 (8,5 \%) fueron secundarios a una obstrucción mecánica extrínseca.

De otro lado, en 15 pacientes $(42,86 \%)$ hubo un compromiso trombótico de las 3 venas suprahepáticas, y en 11 casos $(31,4 \%)$ se presentó trombosis tanto en las venas suprahepáticas como en la vena cava inferior. Además, en 7 casos (20\%) hubo un compromiso aislado de alguna de las venas suprahepáticas y solo en $2(5,7 \%)$ se observó un compromiso exclusivo de la vena cava inferior (Tabla 1 ).

En cuanto al plan de manejo, 32 pacientes (91,4 \%) recibieron anticoagulación plena. Respecto al manejo invasivo para mejorar el drenaje hepático, en la aproximación inicial solo 5 pacientes fueron conducidos a procedimientos: 2 con angioplastia y endoprótesis, 1 con TIPS y un caso de TOH (Figura 2).

De los pacientes con deterioro clínico o ausencia de respuesta con la primera línea de manejo, 17 (48,5\%) recibieron procedimientos invasivos, mientras que $8(22,8 \%)$ fueron conducidos a angioplastia y endoprótesis. Además, 5 pacientes $(14,29 \%)$ requirieron TIPS y 4 fueron llevados a TOH (Figura 2). Es importante mencionar que las alternativas de tratamiento fueron definidas por el grupo de hepatología y progresaron en complejidad según el éxito de recanalización y descompresión. Como hallazgo importante, de los 6 pacientes que se llevaron a TIPS, ninguno requirió trasplante hepático.

Respecto a la sobrevida de los pacientes atendidos en el servicio de hepatología, se documentaron 8 muertes (22,8\%), de las cuales solo 3 fueron atribuidas directamente al BCS. En los escenarios restantes, las muertes fueron secundarias a la progresión de las condiciones de base. 


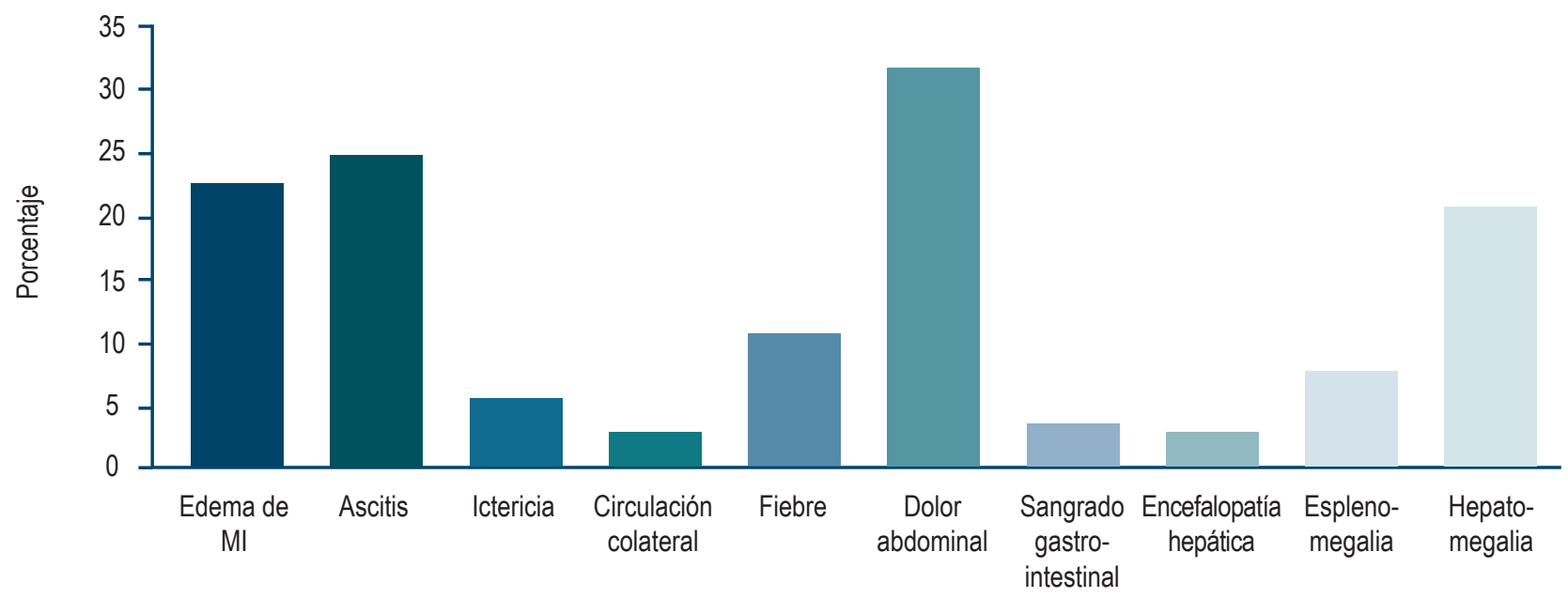

Manifestaciones clínicas

Figura 1. Síntomas clínicos. MI: miembros inferiores.

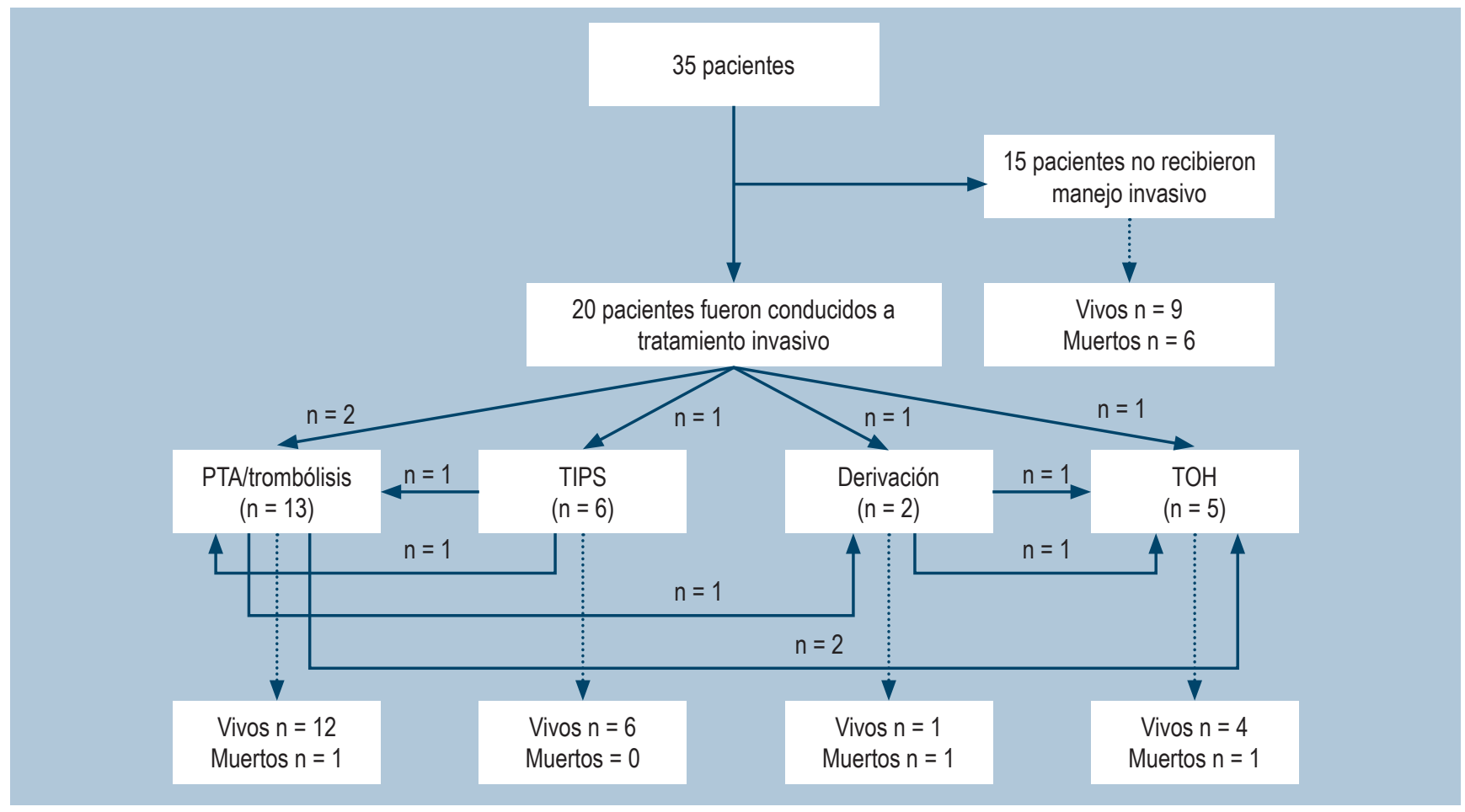

Figura 2. Flujograma de tratamiento. Inicialmente, 1 paciente fue remitido a angioplastia y después recibió trombólisis. PTA: angioplastia transluminal percutánea (Percutaneus Transluminal Angioplasty); TIPS: derivación portosistémica intrahepática transyugular (Transjugular Intrahepatic Portosystemic Shunt); TOH=Trasplante ortotópico hepático.

\section{DISCUSIÓN}

Esta es una de las series más grandes de pacientes con diagnóstico de BCS publicada en nuestro país, y los resultados encontrados son importantes para resaltar. En primer lugar, documentamos que esta condición afecta principalmente a pacientes jóvenes con un promedio de edad que rodea la cuarta década de vida, y que no tiene una preponderancia 
por el género. Dichos hallazgos son comparables con las otras series (4). En nuestro país, ya se habían descrito estas características en reportes de casos (5).

De forma llamativa, durante la formación médica siempre se ha mantenido la idea de que el BCS es una condición de presentación aguda y dramática. Esta forma de presentación solo corresponde a un tercio de los casos (34,29\%); la gran mayoría se manifestaron de forma subaguda o crónica. En nuestra serie, los síntomas cardinales de la presentación clínica, como se ha descrito en la semiología clásica (5), son dolor abdominal $(91,4 \%)$, ascitis $(71,4 \%)$ y hepatomegalia $(60 \%)$. Dichas condiciones siguen teniendo validez en el ejercicio diagnóstico.

Los sitios de obstrucción al tracto de salida hepático corresponden de forma más frecuente al compromiso de las venas suprahepáticas $(42,8 \%)$, o en conjunto con la vena cava inferior $(31,4 \%)$. Son menos habituales las trombosis aisladas de la vena (cava inferior aislada o una vena hepática sola), y en general la gran mayoría de los casos $(91,4 \%)$ responden a causas primarias de trombosis; fueron muy raras las obstrucciones extrínsecas (6).

Como se ha documentado en series previas, el BCS está asociado a trombofilias, ya sean primarias o adquiridas, y a síndromes mieloproliferativos (6). En esta serie, solo en 5 casos no fue posible reconocer la causa de la trombosis y algunos pacientes tenían múltiples etiologías. Sin embargo, al tratarse de un trabajo retrospectivo, no podemos garantizar que a todos los pacientes se les hubiera realizado el mismo protocolo de búsqueda de trombofilia, o que las muestras de laboratorio hubiesen sido tomadas de forma adecuada con respecto al inicio de la anticoagulación.

Así como se ha planteado y demostrado en los trabajos más actuales, definitivamente el tratamiento que logra cambiar el pronóstico de los pacientes es aquel en el cual se consigue la descompresión y la recanalización del tracto de salida hepático (7). En esta serie, 20 pacientes fueron conducidos a procedimientos invasivos y progresaron en complejidad, según la respuesta clínica y bioquímica. No podemos decir que esta intervención se realizó con base en el protocolo de paso a paso, validado por la red europea para el manejo de las enfermedades vasculares del hígado (8). Se trató, más bien, de un ejercicio de juicio clínico metódico y de utilizar las herramientas que teníamos disponibles para cada momento.

A partir de 2013, hemos sido más rigurosos al incluir los puntajes de riesgo para predecir el éxito terapéutico y al valorar la respuesta de forma más temprana, a fin de no retardar el uso de medidas invasivas definitivas que mejoren el pronóstico (9). Aun así, el hallazgo más importante del tratamiento es el excelente resultado logrado en los pacientes a quienes fue posible conducir a TIPS. A pesar de ser pocos casos, este resultado concuerda con las publicaciones actuales $(8,10)$.

Somos conscientes de que una gran parte de las alternativas de tratamiento aquí expuestas no están disponibles en todos los niveles de atención de nuestro sistema de salud. Con seguridad, la mayoría de los centros solo tendrán disponible la anticoagulación, e incluso la trombólisis. No obstante, es evidente, tanto en esta serie como en los grandes reportes a nivel mundial, que hoy en día el éxito del manejo de los pacientes con BCS depende de las intervenciones tempranas, así como de la utilización de puntajes que puedan predecir el éxito de las terapias, y de la selección de los pacientes que, de antemano, se sabe que van a fracasar con las terapias convencionales. Estos pacientes rápidamente requieren de la descongestión hepática, a través de la TIPS o el trasplante hepático.

Las limitaciones de este estudio son las propias de una serie retrospectiva, en la que se reporta la experiencia de un único centro. Además, la disponibilidad de pruebas diagnósticas y terapéuticas no fue la misma para todos los pacientes en el período de tiempo seleccionado. Los planes de manejo no estuvieron estandarizados con respecto a la anticoagulación y la trombólisis. Asimismo, en la fase aguda, la mayoría de los pacientes recibieron heparinas de bajo peso molecular y trombolíticos convencionales.

Sin embargo, a partir de los resultados de esta serie podemos sacar algunas conclusiones. El BCS continúa siendo una condición poco frecuente, que afecta la salud de personas jóvenes, tanto hombres como mujeres, con una presentación clínica heterogénea. En la mayoría de los casos, este síndrome ocurre por una causa desencadenante clara, y predominan las trombofilias y los síndromes mieloproliferativos. El uso de medidas terapéuticas invasivas, especialmente de la selección temprana de los pacientes que se benefician de TIPS, ha cambiado la historia natural y el pronóstico de este grupo de pacientes.

\section{Apoyo financiero}

Este trabajo no contó con apoyo financiero de ninguna entidad. 


\section{REFERENCIAS}

1. Valla DC. The diagnosis and management of the Budd-Chiari syndrome: consensus and controversies. Hepatology. 2003;38(4):793-803. http://doi.org/10.1053/jhep.2003.50415

2. DeLeve LD, Valla DC, Garcia-Tsao G; American Association for the Study Liver Diseases. Vascular disorders of the liver. Hepatology. 2009;49(5):1729-1764. http://doi.org/10.1002/hep.22772

3. Xue H, Li YC, Shakya P, Palikhe M, Jha RK. The role of intravascular intervention in the management of BuddChiari syndrome. Dig Dis Sci. 2010;55(9):2659-2663. http://doi.org/10.1007/s10620-009-1087-7

4. Darwish Murad S, Plessier A, Hernandez-Guerra M, Fabris F, Eapen CE, Bahr MJ, Trebicka J, Morard I, Lasser L, Heller J, Hadengue A, Langlet P, Miranda H, Primignani M, Elias E, Leebeek FW, Rosendaal FR, Garcia-Pagan JC, Valla DC, Janssen HL; EN-Vie (European Network for Vascular Disorders of the Liver). Etiology, management, and outcome of the Budd-Chiari syndrome. Ann Intern Med. 2009;151(3):167-75. http://doi.org/10.7326/0003-4819-151-3-200908040-00004

5. Ramírez AC, Correa S, Espinoza YP, Marín JI, Muñoz O, Santos O, Hoyos S, Guzmán C, Mena A, Restrepo JC. Síndrome de Budd Chiari: Reporte de tres casos y revisión de la literatura. Rev Col Gastroenterol. 2014;29(3):281-284.

6. Okuda H, Yamagata H, Obata H, Iwata H, Sasaki R, Imai F, Okudaira M, Ohbu M, Okuda K. Epidemiological and clinical features of Budd-Chiari syndrome in Japan. J Hepatol. 1995;22(1):1-9.

http://doi.org/10.1016/0168-8278(95)80252-5
7. Attwell A, Ludkowski M, Nash R, Kugelmas M. Treatment of Budd-Chiari syndrome in a Liver Transplant Unit, the role of transjugular intrahepatic porto-systemic shunt and liver transplantation. Aliment Pharmacol Ther. 2004;20(8):867-73. https://doi.org/10.1111/j.1365-2036.2004.02190.x

8. Seijo S, Plessier A, Hoekstra J, Dell'era A, Mandair D, Rifai K, Trebicka J, Morard I, Lasser L, Abraldes JG, Darwish Murad S, Heller J, Hadengue A, Primignani M, Elias E, Janssen HL, Valla DC, Garcia-Pagan JC; European Network for Vascular Disorders of the Liver. Good longterm outcome of Budd-Chiari syndrome with a step-wise management. Hepatology. 2013;57(5):1962-8. http://doi.org/10.1002/hep.26306

9. Qi X, Ren W, Wang Y, Guo X, Fan D. Survival and prognostic indicators of Budd-Chiari syndrome: A systematic review of 79 studies. Expert Rev Gastroenterol Hepatol. 2015;9(6):865-875. https://doi.org/10.1586/17474124.2015.1024224

10. García-Pagán JC, Heydtmann M, Raffa S, Plessier A, Murad S, Fabris F, Vizzini G, Gonzales Abraldes J, Olliff S, Nicolini A, Luca A, Primignani M, Janssen HL, Valla D, Elias E, Bosch J; Budd-Chiari Syndrome-Transjugular Intrahepatic Portosystemic Shunt Group. TIPS for Budd-Chiari syndrome: long-term results and prognostics factors in 124 patients. Gastroenterology. 2008;135(3):808-15. http://doi.org/10.1053/j.gastro.2008.05.051 